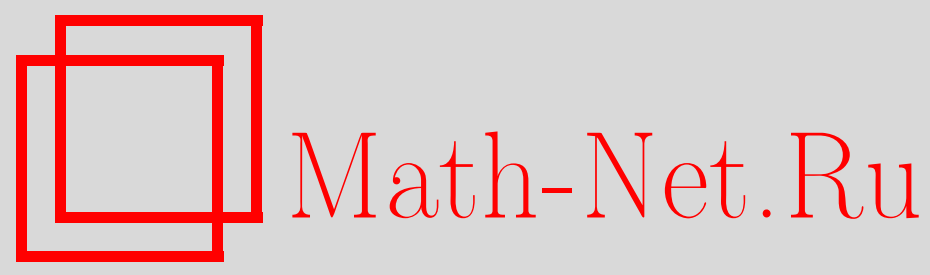

В. И. Малыхин, Борелевская разложимость компактов и их подпространств, Матем. заметки, 1998, том 64, выпуск 5, 701-712

DOI: https://doi.org/10.4213/mzm1446

Использование Общероссийского математического портала Math-Net.Ru подразумевает, что вы прочитали и согласны с пользовательским соглашением http://www . mathnet.ru/rus/agreement

Параметры загрузки:

IP : 3.82 .47 .9

26 апреля 2023 г., 11:06:27 


\section{БОРЕЛЕВСКАЯ РАЗЛОЖИМОСТЬ КОМПАКТОВ И ИХ ПОДПРОСТРАНСТВ}

\section{В.И. Малыхин}

Рассматривается вопрос о наличии в тихоновских кубах и их борелевских подпространствах, в диадических компактах дизъюнктных плотных (борелевских) подмножеств. Поставлено несколько проблем.

Библиография: 13 названий.

0. Введение. 100 лет назад в Математике разразился Великий Кризис, связанньй с обнаружением парадоксов в основаниях математики. Парадоксы показали необходимость ограничения некоторых логических рассуждений, способов образования новых множеств. Часть математиков потребовала ревизии многих математических конструкций и построений, связанных прежде всего с аксиомой выбора. Развитая в этом направлении часть математики впоследствии получила название конструктивной. В широком смысле этого слова в такой математике рассматриваются объекты и применяются методы рассуждений несколько ограничительные по сравнению с “обычной” классической математикой. Такой подход давно, но не очень широко применяется и в общей топологии. В данной заметке предпринята попытка такого подхода в исследовании разложимости топологических пространств.

1. "Неклассические" направления в теории разложимости топологических пространств. В 1943 г. Е. Хьюитт [1] назвал пространство разложсимым, если в нем есть два дизъюнктных плотных подмножества, и неразложсимым в противном случае. Хьюитт, чуть позже М. Катетов [2], другие топологи доказали разложимость многих классов пространств (все рассматриваемые пространства предполагаются не имеюшими изолированных точек): компактов, пространств счетного характера и т.п. K настоящему времени получено много интересных и красивых результатов: Н. В. Величко [3] и Е. Г. Пыткеев [4] доказали разложимость и максимальную разложимость $k$-пространств. В 1994 г. В. Комфорт (см. [5]) доказал разложимость всякого тихоновского счетно компактного пространства. В 1976 г. автор [6] построил на счетной бесконечной булевой групше (вся из элементов второго порядка) неразложимую хаусдорфову групповую топологию, а совсем недавно Комфорт и Ван Милл [7] доказали, что абелева хаусдорфова группа разложима, если в ней множество элементов второго порядка конечно.

Работа выполнена при финансовой поддержке Российского фонда фундаментальных исследований, грант № 96-01-01619. 
Однако постепенно, с исчерпыванием классической тематики выявилась необходимость и возможность более ограничительного подхода к понятию разложимости. Сказалась и некоторая накапливающаяся неудовлетворенность классической теорией разложимости. Эта неудовлетворенность простирается в двух направлениях: во-первых, сами плотные подмножества в большинстве случаев весьма неконструктивны, так как получаются в результате трансфинитных построений; и во-вторых, насчет разложимости рассматриваются самые общие топологические пространства и неразложимые топологии также строятся по трансфинитной индукции.

На первом направлении можно попытаться изменить само понятие разложимости и считать разложимыми (в новом уточненном смысле) только такие пространства, которые имеют пару дизъюнктных плотных подмножеств сравнительно простой природы, например, борелевских (т.е. принадлежащих $\sigma$-кольцу или $\sigma$-алгебре подмножеств, порожденных открытьми или замкнутьми подмножествами рассматриваемогопространства) - и тогда говорят о борелевской разложимости (идея борелевской разложимости была выдвинута С. Ватсоном во время визита автора в Торонто летом 1993 г.).

Может быть, первая работа в этом направлении - это работа Дж. Сидера [8]. Более продвинутой в этом направлении является совместная работа [9] Р. Хименеса и автора. В данной заметке рассматривается борелевская разложимость некоторых классов топологических пространств: компактов, в частности, совершенно нормальных, диадических компактов, пространств полных по Бэру.

На втором направлении можно попытаться рассматривать не всевозможные топологические пространства, а пространства сравнительно простой природы, например, борелевские подмножества тихоновских кубов. Все-таки такие подпространства имеют некоторую "конструктивную” основу; во всяком случае, их топология определяется не трансфинитным построением, а некоторым другим способом. Немаловажно также, что борелевских подпространств (т.е. топологических пространств, гомеоморфных "каноническим" борелевским подмножествам) намного меньше, чем всех топологических подпространств. Так, легко видеть, что в тихоновском кубе веса $n$ всевозможных подпространств $\exp (\exp n)$, в то время как борелевских подпространств только $\exp n$.

Есть и третье "неклассическое" направление в теории разложимости: исследовать "хорошие" (или “хорошо устроенные") топологические пространства на предмет нахождения в них "хороших" плотных дизъюнктных подмножеств.

В данной заметке рассматриваются "хорошие" пространства - борелевские и $A$-подмножества, подпространства тихоновских кубов на предмет нахождения в них как произвольных плотных подмножеств, так и плотных борелевских же подмножеств.

Через [·] и Int обозначаются операторы взятия замькания и внутренности подмножеств топологического пространства.

2. Борелевская разложимость полных по Бэру пространств. Пусть $(X, \tau)-$ топологическое пространство, $\mathscr{B}$ - какое-то семейство его подмножеств. Тогда пространство называется $\mathscr{B}$-разложимым, если в нем имеется два дизъюнктных плотных подмножества, принадлежащих семейству $\mathscr{B}$ каждое. Если таких дизъюнктных подмножеств найдется $k$ штук, то будем говорить, что пространство $k \mathscr{B}$-разложимо; $\Delta(\tau)$-разложимость назьвается максимальной $\mathscr{B}$-разложимостью. (Напомним, что $\Delta(\tau)=\min \{|V|: \varnothing \neq V \in \tau\}$ и назьвается дисперсионным характером топологии $\tau$ или пространства $(X, \tau)$.) Такое общее понятие разложимости введено в [9]. Оно есть прямое обобщение обычной разложимости топологических пространств, когда $\mathscr{B}$ есть семейство всех подмножеств исследуемого пространства. Если же $\mathscr{B}$ есть $\sigma$-алгебра бо- 
релевских подмножеств, порожденная открытыми (или замкнутыми, что в случае алгебры одно и то же) подмножествами, то говорят о борелевской разложимости, а если это $\sigma$-алгебра подмножеств, обладающих свойством Бэра (т.е. представимых в виде $V \triangle N$, где $V$ открыто, $N$ - множество первой категории и $\triangle$ - операция симметрической разности), то говорят о бэровской разложимости. Представление пространства в виде соответствующего дизъюнктного семейства плотных подмножеств будем называть разложсением, а сами плотные подмножества - слагаемы.ми разложения.

Заметим теперь, что слагаемые борелевского разложения, являясь борелевскими множествами, обладают свойством Бэра; следовательно, борелевская разложимость влечет бэровскую разложимость, а бэровская неразложимость влечет неразложимость борелевскую. Вопрос о совпадении или различии этих видов разложимости остается открытым, хотя оба вида разложимости совпадают для всюду полных по Бэру пространств (см. далее следствие 2).

Первые результаты относительно бэровской и борелевской разложимости получены в [9] (см. теоремы 1,2 и следствие 1 далее).

Теорема 1. Метризуемое пространство максимально борелевски, тем более, максимально бәровски разложимо.

ТЕОрема 2. Пусть каждое подмножество первой категории нигде не плотно в пространстве. Тогда пространство бэровски, тем более, борелевски неразложимио.

СлЕдСТВИЕ 1. Следующие пространства бэровски, тем более, борелевски неразложсимы:

а) стоун-чеховский нарост счетного бесконечного дискретного пространст$8 a$;

б) стоуновское пространство булевой алгебры измеримых по Лебегу подмножеств обичного отрезка действительных чисел;

в) континуум Суслина.

ПоЯСНЕНИЕ К ВЫВОДУ СЛЕДСТВИЯ 1 ИЗ ТЕОРЕМЫ 2. В первых двух пространствах каждое подмножество первой категории нигде не плотно в пространстве, а континуум Суслина становится таковым после удаления из него всех сепарабельных интервалов, если они в нем имелись.

Из следствия 1 видно, что бэровская и борелевская разложимость разительно отличаются от классической - ведь в смысле последней любой компакт максимально разложим!

Обобщим теорему 2.

TЕОрема 3. Пусть в пространстве есть непустое открытое множество, в котором каждое подмножество первой категории нигде не плотно. Тогда пространство бәровски, тем более, борелевски неразложимо.

ДокАЗАТЕЛьство. Пусть $V$ - указанное непустое открытое множество. Докажем, что всякое его подмножество $W$ со свойством Бэра, не являющееся нигде не плотньм, имеет непустую внутренность. Легко видеть, что отсюда вытекает теорема 3. Итак, $W=E \triangle N$, где $E$ открыто, а $N$ первой категории. Тем самьг, $W=\left(E \backslash N_{1}\right) \cup N_{2}$, где $N_{1}, N_{2}$ - также множества первой категории. Но $E$ не может быть пустым, ибо тогда 
$W=N_{2}$ и, значит, $W$ нигде не плотно, так как $N_{2}$ первой категории. Если же $E \neq \varnothing$, то Int $W \neq \varnothing$, поскольку $N_{1}$ нигде не плотно.

Теперь дополним теоремы 2,3 , но предварительно напомним, что пространство называется всюду полным по Бәру, если любое множество первой категории имеет пустую внутренность (эквивалентно - пересечение любого счетного семейства плотных открытых множеств всюду плотно).

ТЕорема 4. Для всюду полного по Бәру пространства верно только одно из двух следующих взаимоисключающих утверждении:

А) в пространстве есть непустое открытое множество, в котором каждое подмножество первой категории нигде не плотно; тогда пространство бэровски, тем более, борелевски неразложимо;

Б) в пространстве есть плотное $F_{\sigma}$-подмножество первой категории; тогда пространство борелевски разложимо, так как дополнение к указанному $F_{\sigma}$-подмнохеству, являясь $G_{\delta}$-множеством, также плотно в пространстве, потому что это пространство всюду полно по Бәру (отметим также, что в этом борелевском разложении слагаемые принадлежат к низиим классам борелевской иерархии: одно есть $F_{\sigma}$-множество, а другое $G_{\delta}$-мнохсество).

СлЕДСТВИЕ 2. Всюду полное по Бәру пространство или борелевски разложимо, а тогда и бәровски разложимо, или бәровски неразложимо, а тогда и борелевски неразложимо; другими словами, оба вида разложимости совпадают для таких пространств.

Теорема 4 в какой-то степени решает вопрос Ватсона, который для борелевской разложимости желал иметь слагаемые наинизших классов борелевской иерархии.

ДоКАЗАТЕЛЬСТво ТЕОРЕмЫ 4 . Пусть для пространства $X$ случай А) не имеет места; тогда найдется дизъюнктное семейство $\mathscr{E}$ открытых множеств такое, что $Є \mathscr{E}$ плотно в пространстве и в каждом $E \in \mathscr{E}$ плотно некоторое множество первой категории $F_{E}=\bigcup\left\{F_{i}^{E}: i \in \omega\right\}$, являющееся относительным $F_{\sigma}$-множеством (т.е. каждое $F_{i}^{E}$ замкнуто лишь в $E)$. Пусть $F_{i}=\left[\cup\left\{F_{i}^{E}: E \in \mathscr{E}\right\}\right]$. Тогда $S=\bigcup\left\{F_{i}: i \in \omega\right\}$ есть $F_{\sigma}$-множество первой категории в $X$, плотное во всем пространстве. Если пространство всюду полно по Бэру, то каждое $E \backslash F_{E}$ плотно в $E$; поскольку $E \cap F_{i}=F_{i}^{E}$ для каждых $E \in \mathscr{E}$ и $i \in \omega$, то $E \cap S=F_{E}$ для каждого $E \in \mathscr{E}$. Тогда $X \backslash S$ есть плотное во всем пространстве $G_{\delta}$-множество.

Напомним, что нормальное пространство назьвается совершенно нормальным, если всякое замкнутое его подмножество есть пересечение счетного семейства открытых подмножеств. Совершенно нормальные компакты весьма близки к метрическим. По теореме 1 метризуемое пространство максимально борелевски разложимо, а вот уже с совершенно нормальными компактами дело обстоит иначе.

СлЕдСТВИЕ 3. Утверждение о борелевской разложимости совершенно нормального компакта неразрешимо в ZFC.

Действительно, в ZFC невозможно доказать такое утверждение, ибо континуум Суслина бэровски, тем более борелевски, неразложим по следствию 1 , а ведь он совершенно нормален и его существование совместно с ZFC (см. [10, с. 93-101]). С другой стороны, в 
предположении аксиомы Мартина, соединенной с отрицанием континуум-гипотезы, всякий совершенно нормальньй компакт $X$ сепарабелен (см. [10, с. 101-110]). Пусть $S$ - его

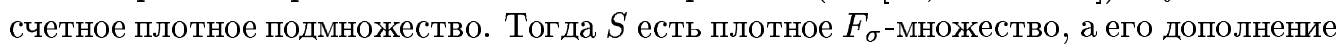


компакт полон по Бэру.

Для полного понимания следствия 3 отметим специально, что бэровская неразложимость, тем более борелевская неразложимость, континуума Суслина есть следствие только его внутренних свойств (см. следствие 1) и никак не связаны с какими-нибудь специфическими свойствами модели, в которой он существует. Кроме того, напомним, что в предположении аксиомы Мартина, соединенной с отрицанием континуум-гипотезы, континуумов Суслина не существует.

ТЕорема 5. В диадическом компакте есть дизбюнктное семейство мощности $\aleph_{1}$ плотных $F_{\sigma}$-подмножсеств, так что он $\aleph_{1}$ борелевски разложим, причем слагаемые имеют тип $F_{\sigma}$.

(В [9] было доказано, что любой диадический компакт имеет два плотных дизъюнктных подмножества: одно $F_{\sigma}$-множество, другое $G_{\delta}$-множество.)

ДокАЗАтЕльство. Так как диадический компакт удовлетворяет условию Суслина, найдется максимальное дизъюнктное семейство $\mathscr{E}$ замкнутых $G_{\delta}$-множеств с пустой внутренностью каждое. Из максимальности семейства вытекает, что его объединение плотно в пространстве. Согласно результату Б.А. Ефимова [11] в диадическом компакте любое семейство $\mathscr{P} G_{\delta}$-множеств имеет счетное подсемейство $\mathscr{P}^{\prime}$ такое, что $\cup \mathscr{P} \subseteq\left[\cup \mathscr{P}^{\prime}\right]$. Найдем такое счетное подсемейство $\mathscr{E}_{0}$ для семейства $\mathscr{E}$. Ясно, что $\cup \mathscr{E}_{0}$ есть плотное в пространстве $F_{\sigma}$-множество. Теперь, так как всякий компакт всюду полон по Бэру, $\cup(\mathscr{E} \backslash \mathscr{E} 0)$ плотно в пространстве. Выделим из него опять-таки счетное подсемейство $\mathscr{E}_{1}$ такое, что $\cup\left(\mathscr{E} \backslash \mathscr{E}_{0}\right) \subseteq\left[\cup \mathscr{E}_{1}\right]$. Следовательно, $\cup \mathscr{E}_{1}$ плотно во всем компакте. Очевидно, что можно продолжить процесс по всем счетньп ординалам. В результате получим желаемое семейство мошности $\aleph_{1}$ дизъюнктных плотных в пространстве $F_{\sigma \text {-множеств. }}$

\section{ГипотезА 1. Диадические компакты максимально борелевски разложимы.}

Отметим теперь, что в сепарабельном компакте $X$ без изолированных точек есть два дизъюнктньх плотных борелевских подмножества: одно - это плотное счетное подмно-

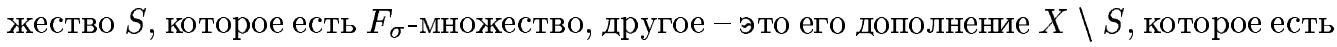
плотное $G_{\delta}$-множество; таким образом, сепарабельньй компакт без изолированных точек борелевски разложим. Однако, возникает следующая

ПроБлЕма 1. Верно ли, что в любом сепарабельном компакте без изолированных точек есть (хотя бы) два дизъюнктных плотных $F_{\sigma}$-множества?

3. Разложимость результатов $A$-операции над открытыми подмножествами тихоновских кубов. Напомним, что такое $A$-операция. Пусть $\omega$ - первьй бесконечньй ординал.

Пусть $\omega^{\omega}-$ множество всех функций из $\omega$ в $\omega$ и $\omega<\omega-$ множество всех функций, определенных на начальных конечных сегментах $[0, n) \subset \omega$ или, что то же самое, $\omega<\omega-$ это множество сужений $\left\{f \mid n: n \in \omega, f \in \omega^{\omega}\right\}$ полных функций из $\omega$ в $\omega$ на начальные конечные сегменты $[0, n) \subset \omega$. 
Пусть $X$ - произвольное множество и $\left\{F_{p}: p \in \omega^{<\omega}\right\}$ - система его подмножеств. Множество $\cup\left\{\cap\left\{F_{f \mid n}: n \in \omega\right\}: f \in \omega^{\omega}\right\}$ назьвается результатом $A$-операции над этой системой.

Теорема 6. Любая сумма $G_{\delta}$-множеств тихоновского куба максимально борелевски разложима, причем ее слагаемые есть $F_{\sigma}$-мнохества.

СледствиЕ 4. Результат А-операчии над открытыми множествами тихоновского куба максимально борелевски разложим, причем слагаемые есть $F_{\sigma-\mathcal{н о -}}$ жествв.

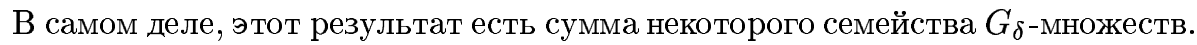

Доказательство теоремы 6 состоит из нескольких предложений.

ПРЕДЛОЖЕНИЕ 1. Тихоновский куб максимально борелевски разложим, причем слагаемые есть $F_{\sigma}$-множества.

ДокАЗАТЕЛЬСТво. Напомним сначала, что б-произведение по базисной точкеэто множество всех точек куба $I^{\alpha}$, отличных от базисной лишь на конечном числе координат. Как легко видеть, каждое $\sigma$-произведение по любой базисной точке плотно в таком кубе. Кроме того, оно есть $F_{\sigma}$-множество. Мощность любого $\sigma$-произведения равна мощности индексного множества; следовательно, различных $\sigma$-произведений в тихоновском кубе $I^{\alpha}$ ровно $\exp \alpha$ и, поскольку различные $\sigma$-произведения дизъюнктны, требуемое доказано.

ПРЕДЛОЖЕНИЕ 2. Всякое $G_{\delta}$-множество тихоновского куба максимально борелевски разложимо, причем слагаемые есть $F_{\sigma}$-множсества.

ДокАЗАТЕльство. В случае куба счетного веса это вытекает из его наследственной сепарабельности, так что рассмотрим куб несчетного веса. Итак, пусть $X$ есть $G_{\delta}$-множество тихоновского куба $I^{\alpha}$ несчетного веса. Тогда найдется счетное подмножество $N \subset \alpha$ и счетное подмножество $S \subset I^{N}$ такие, что $S \times I^{\alpha \backslash N}$ содержится в $X$ и плотно в нем. Как только что доказано, тихоновский куб $I^{\alpha \backslash N}$ максимально борелевски

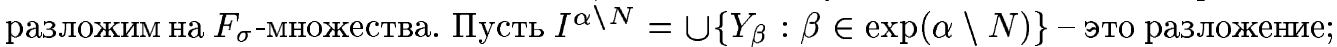
тогда $F_{\sigma}$-подмножества $S \times Y_{\beta}, \beta \in \exp (\alpha \backslash N)$, дизъюнктны и плотны в $X$, что и требовалось доказать.

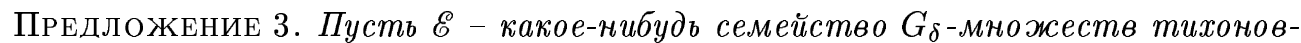
ского куба. Тогда существует дизбюнктное семейство $\mathscr{E}^{\prime} G_{\delta}$-множсеств, вписанное в $\mathscr{E}$, такое, что $\cup \mathscr{E} \subseteq[\cup \mathscr{E}]$.

ДокАЗАТЕЛЬСТво. Построение семейства $\mathscr{E} '$ проведем по трансфинитной индукции. Пусть $\mathscr{E}=\left\{E_{\beta}: \beta<\theta\right\}$. Положим $\mathscr{E}_{0}^{\prime}=\left\{E_{0}\right\}$. Теперь предположим, что для всех

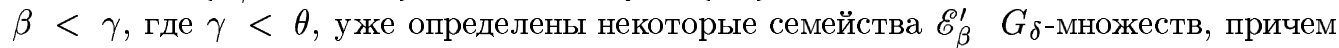
$\cup \mathscr{E}_{\beta}^{\prime} \subseteq E_{\beta}$, семейство $\mathscr{E}_{\gamma}^{*}=\cup\left\{\mathscr{E}_{\beta}^{\prime}: \beta<\gamma\right\}$ дизъюнктно и $\cup\left\{E_{\beta}: \beta<\gamma\right\} \subseteq\left[\cup \mathscr{E}_{\gamma}^{*}\right]$. Теперь найдем счетное дизъюнктное семейство $\mathscr{E}_{\gamma}^{\prime}$ (может быть, пустое) $G_{\delta}$-множеств такое, что $\cup \mathscr{E}_{\gamma}^{\prime} \subseteq E_{\gamma} \backslash\left[\cup \mathscr{E}_{\gamma}^{*}\right]$ и $E_{\gamma} \subseteq\left[\left(\cup \mathscr{E}_{\gamma}^{*}\right) \cup\left(\cup \mathscr{E}_{\gamma}^{\prime}\right)\right]$ По завершении описанного процесса можно убедиться, что семейство $\left\{\mathscr{E}_{\gamma}^{\prime}: \gamma<\theta\right\}$ искомое.

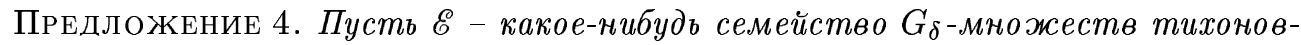
ского куба. Тогда существует счетное подсемейство $\mathscr{E}^{\prime} \subseteq \mathscr{E}$ такое, что $\cup \mathscr{E} \subseteq$ $\left[\cup \mathscr{E}^{\prime}\right]$. 
Это простое следствие уже упомянутого вьше аналогичного утверждения Ефимова, относящегося к $G_{\delta}$-множествам произвольного диадического компакта.

Теперь, используя предложения $1-4$, завершим доказательство теоремы 6 . Заметим сразу же, что в случае куба счетного веса любое его подпространство максимально борелевски разложимо на $F_{\sigma}$-множества, что вытекает из его наследственной сепарабельности, так что рассмотрим куб несчетного веса.

Пусть $\mathscr{E}$ - какое-нибудь семейство $G_{\delta}$-множеств тихоновского куба $I^{\alpha}$ несчетного

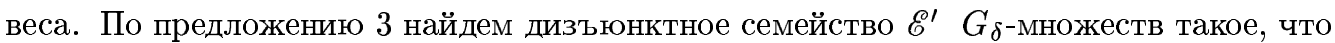
$\cup \mathscr{E}^{\prime} \subseteq \cup \mathscr{E}$ и $\cup \mathscr{E} \subseteq\left[\cup \mathscr{E}^{\prime}\right]$. По предложению 4 найдем счетное подсемейство $\mathscr{E} \prime \subseteq \mathscr{E}^{\prime}$ такое, что $\cup \mathscr{E} \prime \subseteq[\cup \mathscr{E} \prime \prime]$. По предложению 2 в каждом $E \in \mathscr{E}^{\prime \prime}$ найдется дизъюнктное семейство $\left\{\bar{F}_{\beta}^{E}: \beta \in \exp \alpha\right\}$ мощности $\exp \alpha$ плотных в $E F_{\sigma}$-подмножеств. Пусть $F_{\beta}=\bigcup\left\{F_{\beta}^{E}: E \in \mathscr{E}^{\prime \prime}\right\}$ для каждого $\beta \in \exp \alpha$. Тогда легко убедиться, что $\left\{F_{\beta}: \beta \in\right.$ $\exp \alpha\}$ есть дизъюнктное семейство $F_{\sigma}$-подмножеств, плотных в $\cup \mathscr{E}$ и, следовательно, $\cup \mathscr{E}$ содержит максимально много дизъюнктных плотных $F_{\sigma}$-подмножеств.

Заметим, что любая сумма $G_{\delta}$-множеств тихоновского куба $I^{\alpha}$ имеет вес не более $\alpha$, а дисперсионный характер $\exp \alpha$ и, значит, максимально, т.е. $\exp \alpha$-разложима. Но нами доказано нечто большее - можно найти плотные дизъюнктные $F_{\sigma}$-множества.

4. Разложимость $A$-, $C A$ - и $P C A$-множеств в компактах. Результат $A$-операции над системой замкнутых подмножеств топологического пространства назьвается $A$-множеством в нем.

Исследование борелевских подмножеств из $\sigma$-кольца, порожденного замкнутыми подмножествами тихоновского куба, дает некоторые другие результаты (по сравнению с п. 3).

Действительно, всякое счетное подмножество в таком кубе принадлежит указанному $\sigma$-кольцу, но поскольку существуют счетные тихоновские неразложимые пространства (такие построил еще Хьюитт), стало быть, существуют элементы этого кольца, не разложимые в классическом смысле, тем более борелевски неразложимые.

Но счетный случай частенько оказьвается особым при исследовании борелевских и $A$-множеств, так что следующие результаты представляются вполне естественными.

Теорема 7. Регулярное подпространство несчетного дисперсионного характера, являющееся $A$-мнохеством в счетно компактном $T_{1}$-пространстве, разложимоо.

СЛЕДСТВИЕ 5. Подпространство несчетного дисперсионного характера, являющееся $A$-мнохсеством в регулярном счетно компактном пространстве (в частности, в компакте), разложимо.

Напомним, что $A$-множества включают в себя все элементы $\sigma$-борелиана, порожденного замкнутыми множествами, в частности, каждое $F_{\sigma}$-множество есть $A$-множество. (Борелиан - наименьшее семейство, содержащее данное и замкнутое относительно счетных сумм и пересечений.)

СледСТВИЕ 6. Регулярное б-компактное пространство несчетного дисперсионного характера разложимо.

Такое пространство вполне регулярно, а значит, вкладьвается в тихоновский куб, и там оно есть $A$-множество. 
Перейдем теперь к исследованию более специальных подмножеств топологических пространств. Эти подмножества появились в классической дескриптивной теории множеств.

Напомним, что $C A$-множсества - это дополнения к $A$-множествам. Возьмем теперь произведение двух пространств $X_{1} \times X_{2}$, и пусть $\pi_{1}$ - проекция из этого произведения на $X_{1}$. Пусть $E$ есть $A$-множество в $X_{1} \times X_{2}$. Тогда $T=\pi_{1}\left(X_{1} \times X_{2} \backslash E\right)$ называется $P C A$-множеством, а $X_{1} \backslash T$ назьвается $C P C A$-множеством. Легко видеть, что это множество есть мальй образ $A$-множества $E$, т.е. $X_{1} \backslash T=\left\{x \in X_{1}: \pi_{1}^{-1}(x) \subset E\right\}$. Если оба сомножителя $X_{1}, X_{2}$ компактны, то будем говорить о $P C A$ - и $C P C A$-множествах в компактах.

Теорема 8. Дополнение $\kappa$ финально компактному подпространству регулярного счетно компактного пространства разложимо.

Напомним, что всякое $A$-множество в компакте финально компактно; следовательно, $C A$-множество в компакте есть дополнение к финально компактному подпространству. Так что теорема 8 влечет

СлЕДСТВИЕ 7. СА-множсество в компакте разложимо.

Теорема 9. Пусть Е - финально компактное подпространство регулярного счетно компактного произведения $X_{1} \times X_{2}$. Тогда подпространство $\pi_{1}\left(X_{1} \times X_{2} \backslash E\right)$ разложсимо.

Напомним, что $\pi_{1}$ - проекция из этого произведения на $X_{1}$.

СлЕДСТВИЕ 8. РСА-множество в компактах разложимо.

Приводимые далее примеры придают некоторую завершенность результатам данной части.

ПРИмеР 1. Хаусдорфово неразложимое счетно компактное пространство. Подобный пример построен также О. Павловым. Он использовал другую идею.

ПРимеР 2. Хаусдорфово неразложимое $\sigma$-компактное пространство.

ПРимеР 3. Тихоновское пространство и в нем два $\sigma$-компактных подпространства, разность которых имеет несчетный дисперсионньй характер и неразложима.

Пространство из примера 2 финально компактно. С учетом того, что $A$-множество в компакте также финально компактно, представляется интересной следующая

ПроБлЕмА 2. Разложимо ли регулярное финально компактноепространство несчетного дисперсионного характера?

Отметим, что финально компактная группа несчетного дисперсионного характера максимально разложима (см. [12]).

Подчеркнем, что в проблеме 2 речь идет о неразложимости в классическом смысле, ибо существуют компакты, которые бэровски (тем более борелевски) неразложимы (см. выше п. 2).

ПроБлЕмА 3. Разложимо ли $C P C A$-множество в компактах?

Перейдем теперь к доказательству теорем 7-9 и конструкциям примеров 1-3.

Начнем с определения. 
ОПРЕДЕЛЕНИЕ. Точка $x$ называется lsd-moчкой, если существует множество $M$, точки которого имеют дизъюнктное семейство открытых окрестностей $\mathscr{V}=\left\{V_{m}: m \in V_{m}\right.$, $m \in M\}$, так что $x \in[M]$, но $x \notin \cup \mathscr{V}$. (Название "lsd" образовано начальными буквами слов "а Limit point of a Strongly Discrete subspace". Такой тип точек был введен в [13] под другим названием.)

Следующая лемма взята из [13].

Лемма 1. Если пространство состоит только из lsd-точек, то оно К-разложимо.

\section{Эту лемму использует}

ПРЕДЛОЖЕНИЕ 5. Если всякое непустое открытое подмножество хаусдорфова пространства содерхит бесконечное счетно компактное подмнохсество, то пространство разлохсимо.

ДоказАтЕльство. Докажем сначала, что множество $L$ lsd-точек плотно в таком пространстве. Действительно, пусть $W$ - непустое открытое подмножество; тогда в нем есть бесконечное счетно компактное подмножество $B$. Так как $B$ бесконечно, а пространство хаусдорфово, найдется бесконечное дизъюнктное семейство $\mathscr{V}$ открытых множеств таких, что $V \cap B \neq \varnothing$ для всякого $V \in \mathscr{V}$. Выберем по точке $m(V)$ в каждом таком $V \cap B$. Так как $B$ счетно компактно, множество точек $M=\{m(V): V \in \mathscr{V}\}$ имеет предельную точку $x \in B \subset W$. Это и есть lsd-точка. Итак, доказано, что множество $L$ lsd-точек плотно в рассматриваемом пространстве.

Далее используем

КРИТЕРИЙ НЕРАЗЛОЖИМОСТИ ХЬЮИТТА. Пространство неразложимо, если и только если оно содержит непустое открытое подмножество, в котором всякое плотное в себе подмножество неразложимо.

Теперь покажем, что в нашем пространстве такого открытого подмножества нет, чем и завершим доказательство предложения 5 . Пусть $W$ - какое-нибудь непустое открытое подмножество. Возможны два варианта: $\operatorname{Int}(L \cap W) \neq \varnothing$ или $\operatorname{Int}(L \cap W)=\varnothing$. Но в первом варианте $\operatorname{Int}(L \cap W)$ даже $\aleph_{0}$-разложимо согласно лемме 1 , во втором же варианте $W$ разложимо, потому что дизъюнктные множества $L \cap W$ и $W \backslash L$ имеют пустые внутренности и, значит, оба плотны в $W$.

\section{ДОКАЗАТЕЛЬСТВО ТЕОРЕМЫ 7.}

Лемма 2. Несчетное А-мнохсество в счетно компактном $T_{1}$-пространстве содержит бесконечное замкнутое во всем пространстве подмножество.

ДоказАтельство. Система подмножеств $\left\{F_{p}: p \in \omega^{<\omega}\right\}$ назьвается регулярной, если $F_{s} \supseteq F_{t}$, когда $s \subseteq t$. Известно, что при исследовании $A$-множеств можно ограничиться регулярными системами, так что в дальнейшем соответствуюшие системы будут предполагаться регулярньми. Пусть $A$-множество $E$ получено из системы $\left\{F_{p}: p \in \omega^{<\omega}\right\}$. Объединение всех элементов $F_{p}$, для которых пересечение $E \cap F_{p}$ счетно, также счетно; следовательно, существует $f \in \omega^{\omega}$ такая, что каждое множество $F_{f \mid n}$ несчетно.

Фиксируем какую-либо такую $f$. Заметим, что $\cap\left\{F_{f \mid n}: n \in \omega\right\}$; поэтому если это множество бесконечно, то доказывать нечего - бесконечное замкнутое подмножество в $E$ найдено. Так что пусть это множество конечно. Но тогда найдется последовательность 
кортежей $p_{0} \subseteq p_{1} \subseteq \cdots$ таких, что $f=\bigcup\left\{p_{i}: i \in \omega\right\}$ и $E \cap\left(F_{p_{i}} \backslash F_{p_{i+1}}\right) \neq \varnothing$ для всякого $i \in \omega$. В каждом таком множестве зафиксируем точку $x_{i}$. Теперь легко убедиться, что $\left\{x_{i}: i \in \omega\right\} \cup\left(\cap\left\{F_{f \mid n}: n \in \omega\right\}\right)$ есть бесконечное замкнутое во всем пространстве подмножество, содержащееся в $E$.

Пусть $E$ - какое-нибудь регулярное подпространство несчетного дисперсионного характера, являющееся $A$-множеством в счетно компактном $T_{1}$-пространстве $X$. Покажем, что всякое непустое открытое подмножество $W \subset E$ содержит бесконечное замкнутое во всем пространстве подмножество. Это подмножество, очевидно, счетнокомпактно, и нам остается сослаться на предложение 5.

Итак, пусть $W$-какое-нибудь непустое открытоеподмножество $E$. Пусть $x \in W$. Так как $E$ регулярно, найдется открытая в $E$ окрестность $V$ точки $x$ такая, что $[V]_{E} \subset W$. Но дисперсионный характер $E$ несчетен, а значит, $V$ несчетно, тем более несчетно $[V]_{E}$. Очевидно, $[V]_{E}$ есть $A$-множество в $X$, и вдобавок, несчетное, как только что установлено. Значит, по лемме 2 оно содержит бесконечное замкнутое во всем пространстве подмножество. Но это и требовалось доказать.

ДОКАЗАТЕЛЬСТВО ТЕОРЕМЫ 8.

ЛЕмма 3. Всякая неизолированная точка в дополнении к финально компактному подпространству регулярного счетно компактного пространства является предельной в бесконечном подмножестве әтого дополнения, замкнутом во всем пространстве.

ДокАЗАТЕЛЬСТво. Пусть $E$ - финально компактное подпространство регулярного счетно компактного пространства $X$ и $x$-неизолированная точка в $X \backslash E$. Для всякой точки $e \in E$ фиксируем два открытых множества $V_{e}$ и $W_{e}$ таких, что $x \in V_{e}, e \in W_{e}$ и $\left[V_{e}\right] \cap W_{e}=\varnothing$. Так как $E$ финально компактно, существует счетное семейство $\mathcal{V}$ множеств $\left[V_{e}\right]$, так что $(\cap \mathscr{V}) \cap E=\varnothing$. Пусть $K=\cap \mathscr{V}$. Если $x-$ предельная точка $K$, то все доказано. Иначе, существует открытая окрестность $Q$ точки $x$ такая, что $\{x\}=Q \cap K$. Но тогда $\{x\}=Q \cap\{V:[V] \in \mathscr{V}\}$, т.е. $\{x\}$ есть $G_{\delta}$-множество в регулярном счетно компактном пространстве, значит $x$ имеет счетньй характер. Но тогда, поскольку $x$ - неизолированная точка в $X \backslash E$, ей соответствует сходящаяся последовательность, лежащая в $X \backslash E$.

Теперь докажем непосредственно теорему 8. Пусть $E$ - финально компактное подпространство регулярного счетно компактного подпространства $X$. Докажем, что $X \backslash E$ разложимо (если оно, конечно, не имеет изолированных точек, что предполагается всюду в данной работе, когда речь идет о разложимости). Пусть $W$ - непустое открытое подмножество $X$ и $W \backslash E \neq \varnothing$. В силу регулярности найдется непустое открытое подмножество $V \subset W$ такое, что $[V] \subset W$ и $V \backslash E \neq \varnothing$. Но $[V]$ счетно компактно и $E \cap[V]$ финально компактно. Согласно лемме 3 любая точка из $V \backslash E$ является предельной в некотором бесконечном подмножестве, лежащем в $[V] \backslash E$. Далее осталось сослаться на предложение 5 .

ДОКАЗАТЕЛЬСТВО ТЕОРЕМЫ 9.

ЛЕмма 4. Пусть Е-финально компактное подпространство регулярного счетно компактного произведения $X_{1} \times X_{2}$. Тогда всякая неизолированная точка из $T=\pi_{1}\left(X_{1} \times X_{2} \backslash E\right)$ является предельной в бесконечном подмножсестве из $T$, замкнутом в $X_{1}$. 
ДоказАтЕльство. Ясно, что существует $y \in X_{2}$ такая, что $(x, y) \notin E$. Для всякой точки $e \in E$ фиксируем два открытых подмножества произведения $X_{1} \times X_{2}$ : “прямоугольное" открытое множество $V_{e}=V_{1}^{e} \times V_{2}^{e}$, где $V_{1}^{e}, V_{2}^{e}$ - открытые окрестности точек $x, y$ в пространствах $X_{1}, X_{2}$ соответственно, и открытое множество $W_{e}$ такое, что $e \in W_{e}$ и $\left[V_{e}\right] \cap W_{e}=\varnothing$. Так как $E$ финально компактно, существует счетное семейство $\mathscr{V}$ множеств $\left[V_{e}\right]$, так что $(\cap \mathscr{V}) \cap E=\varnothing$. Пусть $K=\pi_{1}(\cap \mathscr{V})$. Если $x-$ предельная точка $K$, то все доказано. Иначе, существует открытая окрестность $Q$ точки $x$ в $X_{1}$ такая, что $\{x\}=Q \cap K$. Но тогда $\{x\}$ есть $G_{\delta}$-множество в регулярном счетно компактном пространстве $X_{1}$, значит $x$ имеет счетньй характер в нем. Но тогда, поскольку $x-$ неизолированная точка в $T$, к ней имеется сходящаяся последовательность, лежащая в $T$.

Далее доказательство теоремы 9 проходит примерно так же, как и теоремы $8,-\mathrm{c}$ использованием леммы 4 и затем предложения 5.

ПосТРОЕНИЕ ПРИМЕРА 1 . Пусть $X$-какое-нибудь сепарабельное экстремальнонесвязное компактное или счетно компактное регулярное пространство без изолированных точек (например, абсолют отрезка $[0,1])$. Тогда $X$ не имеет сходящихся последовательностей; следовательно, всякое бесконечное замкнутое подмножество в нем несчетно. Пусть $S$ - какое-нибудь счетное плотное подмножество $X$. Обозначим топологию на $X$ через $\nu$, а топологию подпространства на $S$ через $\tau$.

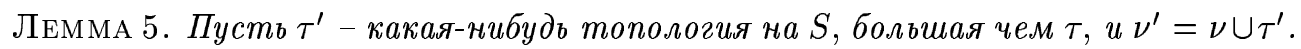
Тогда $\left(X, \nu^{\prime}\right)$ хаусдорфово и счетно компактно.

ДоКАЗАТЕЛЬСТВО. Проверим только счетную компактность, хаусдорфовость очевидна. Ясно, что если $x \in X \backslash S$ и $x \in V \in \nu^{\prime}$, то $V \in \nu$. Пусть $B$ - какое-нибудь бесконечное подмножество $X$. Тогда $[B]_{\nu^{\prime}} \supseteq\left([B]_{\nu} \backslash S\right)$, тем самым, $[B]_{\nu}$ несчетно. Доказана счетная компактность.

Продолжим описание примера 1. Известно, что для всякой топологии на бесконечном множестве существует большая неразложимая топология (также плотная в себе). Пусть $\tau^{+}$- какая-нибудь неразложимая топология на $S$, большая чем $\tau$, и $\nu^{+}=\nu \cup \tau^{+}$. Тогда пространство $\left(X, \nu^{+}\right)$искомое: оно хаусдорфово, неразложимо и счетно компактHO.

ПосТРОЕНИЕ ПРИМЕРА 2. Предварительно сконструируем некоторое необходимое для дальнейшего пространство. Возьмем бэровское пространство веса $\aleph_{1}$, т.е. произведение $\aleph_{0}$ копий дискретного пространства мощности $\aleph_{1}$. Это метрическое пространство, в котором всякое счетное подмножество нигде не плотно. Фиксируем какую-нибудь $\sigma$-дискретную базу $\cup\left\{\mathscr{B}_{i}: i \in \omega\right\}$. Выберем из каждого элемента семейства $\mathscr{B}_{i}$ по точке и обозначим множество выбранных точек $X_{i}$. Выбор можно организовать так, что все различные подмножества $X_{i}, i \in \omega$, дизъюнктны. Пусть $X=\cup\left\{X_{i}: i \in \omega\right\}$. Обозначим топологию подпространства на $X$ через $\rho$. Итак, в $(X, \rho)$ слагаемые $X_{i}$ дизъюнктны, дискретны и замкнуты, более того, точки каждого $X_{i}$ имеют дизъюнктное семейство открытых окрестностей; более того, точки любой суммы $X_{i} \cup X_{k}$ также имеют дизъюнктное семейство открытых окрестностей; и, наконец, каждое счетное подмножество всего $X$ нигде не плотно.

Выше уже упоминалось, что для всякой топологии на бесконечном множестве существует большая неразложимая топология (также плотная в себе). В нашем случае для 
топологии $\rho$ найдется большая неразложимая и несчетного дисперсионного характера топология. Обозначим ее через $\tau$.

Возьмем теперь для всякого $i \in \omega$ какую-нибудь компактификацию $b X_{i}$ дискретного пространства $X_{i}$; обозначим через $\nu_{i}$ топологию на $b X_{i}$. Будем считать множества $\left(b X_{i} \backslash X_{i}\right)$ дизъюнктными. Пусть $Z=\cup\left\{b X_{i}: i \in \omega\right\}$. Рассмотрим на $Z$ топологию $\nu$, определенную так: $V \in \nu$, если и только если $V \cap X \in \tau$ и $V \cap b X_{i} \in \nu_{i}$ для всякого $i \in \omega$. Нетрудно убедиться, что пространство $(Z, \nu)$ искомое: оно хаусдорфово, $\sigma$-компактно, его открытое подпространство $(X, \tau)$ неразложимо, а следовательно, и само $(Z, \nu)$ неразложимо.

ПосТРОЕНИЕ ПРИМЕРА 3. Возьмем пространство $(X, \tau)$ из предыдущего примера. Пусть $\beta X$ - его стоун-чеховское компактное расширение. Пусть для всякого $i \in \omega b X_{i}$ есть замыкание в $\beta X$ дискретного пространства $X_{i}$. Все подпространства $b X_{i} \backslash X_{i}$ попарно дизъюнктны. Пусть $Z=\cup\left\{b X_{i}: i \in \omega\right\}$ и $Y=\cup\left\{b X_{i} \backslash X_{i}: i \in \omega\right\}$. Очевидно, что оба пространства $Z, Y \quad \sigma$-компактны и их разность $Z \backslash Y$ есть пространство $(X, \tau)$, которое неразложимо и имеет несчетньй дисперсионньй характер.

Автор выражает благодарность В. Щиголеву за плодотворное обсуждение некоторых вопросов, относящихся к данной заметке, и рецензенту за многочисленные замечания и предложения, направленные на улучшение стиля и содержания заметки.

\section{СПИСОК ЦИТИРОВАННОЙ ЛИТЕРАТУРЫ}

[1] Hewitt E. A problem of set-theoretic topology // Duke Math. J. 1943. V. 10. P. 309-333.

[2] Катетов M. О топологических пространствах, не содержащих плотных дизъюнктных подмножеств // Матем. сб. 1947. Т. 21. № 1. С. 3-12.

[3] Величко Н. В. К теории разложимых пространств // Матем. заметки. 1976. Т. 19. № 2. C. 209-214.

[4] Пыткеев Е. Г. О максимально разложимых пространствах // Тр. МИАН. 1983. Т. 154. C. 209-213.

[5] Comfort W.W., Garcia-Ferreira S. Resolvability: a selective survey and some new results // Topology Appl. 1996. V. 74. P. 149-167.

[6] Малыхин В.И. Экстремально-несвязные и близкие к ним группы // Докл. АН СССР. 1975. T. 220. №1. C. 27-30.

[7] Comfort W. W., van Mill J. Groups with only resolvable group topologies // Proc. Amer. Math. Soc. 1994. V. 120. P. 687-696.

[8] Ceder J. On maximally Borel resolvable spaces // Rev. Roumaine Math. Pures Appl. 1966. V. 11. P. 89-94.

[9] Jimenez R., Malykhin V.I. Structure resolvability // Comment. Math. Univ. Carolin. 1998. V. 39. № 2. P. 379-388.

[10] Йех Т. Теория множеств и метод форсинга. М.: Мир, 1973.

[11] Ефимов Б. А. Диадические бикомпакты // Тр. ММО. 1965. Т. 14. С. 211-247.

[12] Малыхин В.И. Максимальная разложимость ограниченных групп // Матем. заметки. (в печати).

[13] Sharma P. L., Sharma S. Resolution properties in generalized $k$-spaces // Topology Appl. 1989. V. 29. P. $61-66$.

Государственная академия управления им. С. Орджоникидзе

Поступило

E-mail: matem@acman.msk.su

04.07 .96

Исправленный вариант 03.03.98 